\title{
Two-Dimensional X-Ray Beam Phase Sensing
}

\author{
Sébastien Bérujon, ${ }^{1,2}$ Eric Ziegler, ${ }^{1}$ Roberto Cerbino, ${ }^{3}$ and Luca Peverini ${ }^{1, *}$ \\ ${ }^{1}$ European Synchrotron Radiation Facility, BP-220, F-38043 Grenoble, France \\ ${ }^{2}$ Diamond Light Source, Harwell Science and Innovation Campus, Didcot, Oxfordshire, OX11 ODE, United Kingdom \\ ${ }^{3}$ Università degli Studi di Milano clo LITA, via Fratelli Cervi 93, I-20090 Segrate (MI), Italy
}

(Received 26 November 2010; revised manuscript received 30 August 2011; published 11 April 2012)

We present a new method to analyze quantitatively the wave front of a partially coherent x-ray beam. The technique is based on the use of two-dimensional speckle patterns combined with digital image correlation algorithms and offers a pixel size resolution, a high accuracy, and a reduced sensitivity to mechanical vibrations thanks to a very simple setup. The requirements on transverse and longitudinal coherence are also low. Finally, we show how the method can be used for phase contrast imaging applications by a single sample exposure process.

DOI: 10.1103/PhysRevLett.108.158102

PACS numbers: 87.59. $-\mathrm{e}, 41.50 .+\mathrm{h}$

For both beam metrology and phase contrast imaging purposes, the $\mathrm{x}$-ray phase sensing techniques are the subject of investigations worldwide. Indeed, several x-ray techniques based on the coherence properties of the beam are strongly affected by the local beam characteristics, limiting their performance or degrading their result quality. Thus, the development of tools able to characterize the wave front with accuracy in the order of the x-ray wavelength is required. In parallel, thanks to a high power of penetration, the $\mathrm{x}$-ray photons are able to probe the inner structure of materials. Hard x-ray imaging techniques sensitive to the phase are then the object of special efforts, as they permit to image thick samples or samples presenting a low absorption.

Currently, various techniques such as pencil beam deflectometry, shearing interferometry, Hartmann wave front sensing, and transport of intensity equation (TIE) based methods are available to record and analyze quantitatively the phase of an x-ray beam wave front, or more precisely its derivative. Among them and apart from the basic pencil beam technique, the grating interferometer (GI) [1] is the most widely used advanced instrument due to its accuracy and low requirement on mechanical stability. Nevertheless, the device still suffers one weakness: it is able to measure the wave front gradient in only one direction at a time despite ongoing work [2]. Based on a different principle, the Hartmann sensor [3], does not have this problem: it is able to derive wave front slopes in both directions from a single acquisition. On the other hand, it presents a limited resolution and requires a delicate calibration. TIE based techniques [4] are simple to set but include complex calculations of the data which can suffer from analysis artifacts. Concerning qualitative $\mathrm{x}$-ray phase contrast imaging, the most widespread techniques are presently grating interferometry [5], coherent diffraction imaging [6], and propagation-based techniques [7]. However, they all share the same problem that they require several exposures of the sample to reconstruct one phase image.
The x-ray speckle tracking technique (XST) overcomes these limitations, offering a 2D gradient in a single measurement, a pixel size resolution, and an accuracy equivalent to the one of the instruments mentioned previously. The setup is reduced to a minimum, requiring only a random phase object and a $2 \mathrm{D}$ detector to resolve the high-spatial frequency features contained in the object. A solid membrane, easy to align and with low sensitivity to vibrations, produces a random intensity pattern (speckle) that is static. By recording this random pattern 2 times in planes located at two different distances from the membranes or in the same plane at two different time intervals, the ray paths or their evolution can be tracked using a digital image correlation algorithm (DIC) capable of subpixel accuracy (cf. Pan [8]). The validation of the method has been realized on a synchrotron bending magnet source at beam line BM05 of the ESRF. The fact that it works with a multilayer monochromator proves that the longitudinal coherence requirement is low.

The XST method can be understood as a high-spatial frequency intensity modulation of the wave front using motionless speckle to trace the geometrical path of the light passing through each pixel of the detector. The key idea is represented in Fig. 1: each image subset contains a distinct speckle pattern that acts as a singular marker, and

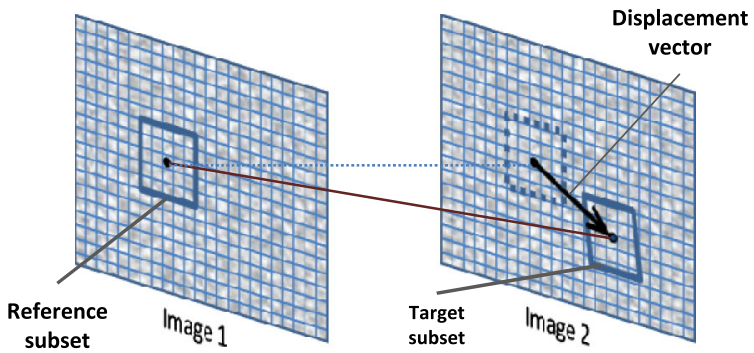

FIG. 1 (color online). DIC principle. The position of a speckle subset of few pixels is tracked from one image to the next using a cross-correlation criterion. 
can be numerically tracked between images taken at different times or in different planes in space.

The use of speckle can be found in many x-ray techniques, such as X-ray photon correlation spectroscopy and coherent diffraction imaging. Contrary to those methods, the XST technique uses the near field speckle also present in the X-ray regime as demonstrated by Cerbino et al. [9]. These speckle grains have the property to not change in size and shape over a distance along the propagation direction that is inversely proportional to the wavelength and therefore is much larger for $\mathrm{x}$ rays than for visible light [10]. With $\mathrm{x}$ rays, the distance between planes (Fig. 1) becomes large enough to use numerical algorithms to follow the speckle trajectories. Indeed, the curvature of the near field speckle trajectories coincide with the curvature of the beam. In practice, the distance $z$ over which speckles of size $d$ can be tracked in depth is not limited to the deep Fresnel region as described by Cerbino $\left(z_{\mathrm{NF}}<d D / \lambda\right.$, where $D$ is the transversal coherence size and $\lambda$ the wavelength), but can go until $z=D^{2} / \lambda$, corresponding to the transition between the Fresnel and far field regimes [11]. Above that distance, the distortion of the tracked subsets becomes too important comparatively to the robustness of the algorithms employed. An additional effect that we can naturally benefit from is the divergence of the x-ray beam: for a beam of divergence $\alpha$, the near field distance is increased by a factor $1 /(1-\alpha d / \lambda)$ and the geometrical magnification of the speckle pattern is small enough to obtain usable correlation coefficients in the numerical algorithms. Hence, the tracking gives excellent results over a range that can vary from several centimeters to many meters, depending on the x-ray energy, the size of the scattering objects, and the beam divergence, as we shall demonstrate with the examples below. To produce a speckle pattern, we used membranes with dephasing objects smaller than the transverse beam coherence but large enough to be resolved with our detector. These membranes, e.g., biological filtering membranes or abrasive paper, are thermally stable under monochromatic x-ray beam.

Two configurations are presented in Fig. 2. In each case, only two speckle images are acquired, allowing the monitoring of the ray path for each pixel using the DIC algorithms. One should understand the fundamental difference between those configurations. The scheme in Fig. 2(a), labeled "differential configuration," uses a reference speckle image plus another image with a phase object introduced in the beam path. When using this setup, the displacement vectors calculated give information about the wave front distortion introduced by the phase object only. The alternative setup in (b), labeled "absolute configuration," uses two images taken at different planes in space. In this latter case, calculation from the two speckle images will give access to the quantitative wave front at one of the image planes, comprising the distortion to the wave front

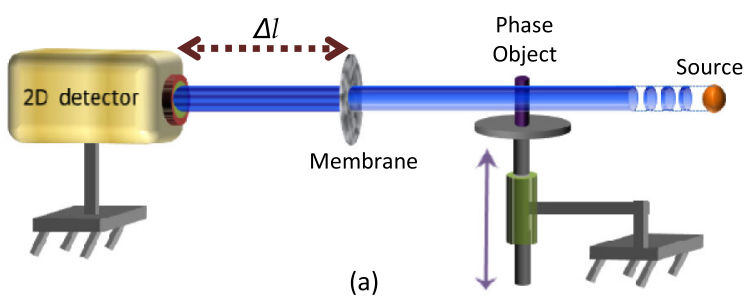

(a)

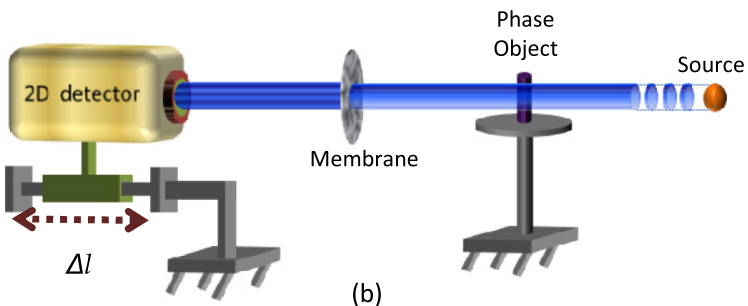

(b)

FIG. 2 (color online). Two possible configurations. In configuration (a), the sample is removed to obtain a reference beam and work differentially, while in configuration (b) two shots are taken at different distances from the membrane to act as a Hartmann sensor, the sample remaining in the beam.

induced by the elements present along the beam path: optical elements, windows, etc. Anyhow, the displacement vector can be compared in both setups to the one used in a Hartmann sensor, i.e., the shifting of a spot from its theoretical position.

The DIC algorithms [8] are usually used to describe the distortion of a material under mechanical strain. The so-called mapping function is commonly employed, to define the motion of a subset centered on a given pixel $P_{\text {ini }}=\left(x_{0}, y_{0}\right)$ in the unitary base $(\mathbf{x}, \mathbf{y})$ :

$$
x_{0}^{\prime}=x_{0}+\xi\left(x_{0}, y_{0}\right), \quad y_{0}^{\prime}=y_{0}+\tau\left(x_{0}, y_{0}\right),
$$

where $\xi\left(x_{0}, y_{0}\right)$ and $\tau\left(x_{0}, y_{0}\right)$ reflect both the translation of the target subset as well as its distortion from the reference subset in $\left(x_{0}, y_{0}\right)$. The DIC scheme is usually a two-step procedure. The first one is the calculations of the displacement vectors of the subsets with pixel accuracy. Those results are then used as an input for the second step in which a subpixel accuracy algorithm is applied. Indeed, these algorithms are able to calculate displacements with a reproducibility of a hundredth of a pixel when providing an initial guess within one pixel radius from the final solution.

Among the algorithms able to calculate the displacement vector of a subset within a one pixel accuracy [8], the zero-normalized cross correlation (ZNCC) is the best choice. The ZNCC criterion evaluates a similarity factor between a subset of $M$ points in the reference image $f$ and a target subset centered on $(\alpha, \beta)$ in a second image $g$ :

$C(\alpha, \beta)=\sum_{x=-M}^{M} \sum_{y=-M}^{M}\left[\frac{[f(x, y)-\bar{f}]\left[g\left(x^{\prime}, y^{\prime}\right)-\bar{g}\right]}{\Delta f \Delta g}\right]$,

where $\bar{f}$ and $\bar{g}$ are the mean value of the subsets and $\Delta f$ and $\Delta g$ their respective standard deviation. Then, for each 
subset around a given pixel, the displacement vector is the one that verifies $\boldsymbol{\nu}=\left(\overrightarrow{P_{\text {ini }}, P_{\max }}\right)$, where $P_{\max }=\left(\alpha_{0}, \beta_{0}\right)$ and $C\left(\alpha_{0}, \beta_{0}\right)=\max _{(\alpha, \beta)} C(\alpha, \beta)$. This correlation criterion being the least sensitive one to spatial and temporal intensity variations in the beam, offers the best choice in terms of robustness. This allows one to perform wave front analysis and imaging downstream a moderately absorbing sample. For the second step, several DIC algorithms with subpixel accuracy have been developed over the last few decades (cf, [8]). In the following experiments, for time consumption issue, we used the MATLAB peak finder algorithm that, like the other algorithms of its class, does not consider the deformation of the subset, treating only the rigid translation of the subset $\boldsymbol{\nu}$ and

$$
\xi\left(x_{0}, y_{0}\right)=\boldsymbol{\nu} \cdot \mathbf{x}=\nu_{x}, \quad \tau\left(x_{0}, y_{0}\right)=\boldsymbol{\nu} \cdot \mathbf{y}=\nu_{y} .
$$

While this is enough to characterize the first order gradient of the wave front using Eq. (4), taking the subsets distortion into account would provide additional information about the second derivative of the wave front, i.e., the wave front curvature [12].

Once the ray paths have been calculated, the phase recovery is done in both configurations, using the relationship linking the local angular deviation of the beam, i.e., the wave front slope, to the gradient of the phase in both directions:

$$
\Theta_{n} \simeq \frac{\nu_{x, y}}{\Delta l}=\frac{\partial W(x, y)}{\partial n}=\frac{\lambda}{2 \pi} \frac{\partial \phi(x, y)}{\partial n}, n \in\{x, y\} .
$$

The attainable accuracy depends directly on the resolution $d_{\text {pix }}$ of the $2 \mathrm{D}$ detector, and on the working distance $\Delta l$ between two image planes or between the membrane and the detector according to the configuration used (Fig. 2). The smallest deviation that can be measured is given by $\Theta_{\text {min }}=d_{\text {pix }} \times \delta_{\mathrm{CCC}} / \Delta l_{\text {max }}$, where $\delta_{\mathrm{CCC}}$ is the pixel accuracy of the cross-correlation criterion. In the experimental setup we used, $\delta_{\mathrm{CCC}} \leq 0.05$ pixel, $\Delta l_{\max }=200 \mathrm{~mm}$, and $d_{\text {pix }}=0.8 \mu \mathrm{m}$, leading to a theoretical accuracy of $\Theta_{\min } \sim 0.1 \mu \mathrm{rad}$, which is already as good as the current GI and Hartmann instrument. A further gain in accuracy of 1 order of magnitude would already be reachable using a smaller pixel size detector, and/or by placing the detector further away from the membrane.

A series of experiments were conducted at beam line BM05 of the ESRF, where the $\mathrm{x}$ rays are produced by a bending magnet on a $6 \mathrm{GeV}$ electron storage ring. The working energy was set to $E=17 \mathrm{keV}(\lambda=0.073 \mathrm{~nm})$ either with a multilayer monochromator $\left(\Delta E / E=10^{-2}\right)$ or with a double flat $\operatorname{Si}(111)$ monochromator $(\Delta E / E=$ $10^{-4}$ ). With the experimental station placed at $40 \mathrm{~m}$ from the source, the transverse coherence length is approximately $9 \mu \mathrm{m}$ horizontally and $25 \mu \mathrm{m}$ vertically [13]. The divergence of the beam is $2.4 \mathrm{mrad}$ horizontally and $180 \mu \mathrm{rad}$ vertically. The beam line specifications are detailed in Ref. [14].
A first simple illustration of the method, using the scheme of Fig. 2(b), is given in Fig. 3: (a) is the reconstruction of the beam wave front after the $\mathrm{Si}(111)$ monochromator. Slits were used to set the beam size to $2.5 \times 2.5 \mathrm{~mm}^{2}$. The field of view of the camera permits us to analyze the beam with a micrometer resolution over several square millimeters. The wave front error from the perfect spherical wave front is shown on Fig. 3(b). We can observe that, over the full aperture, the wave front error is much larger than the wavelength. However, the dephasing over any area of size $9 \times 25 \mu \mathrm{m}^{2}$ (coherence area) is never larger than $\lambda / 2$.

Using the same configuration, we compared the wave front derived from the XST technique when various optical elements were inserted in the beam. The 2D detector was mounted on a precision translation stage with a reproducibility of $1 \mu \mathrm{m}$. The x-ray energy was defined with a multilayer monochromator. An abrasive paper made of $\mathrm{SiC}$ powder with a mean grain size of $5 \mu \mathrm{m}$ was placed at $34 \mathrm{~m}$ from the source, i.e., at $6.5 \mathrm{~m}$ upstream from the detector. For the direct beam measurement, images of the speckle pattern produced by the abrasive paper were imaged at two different positions separated by a distance $\Delta l=200 \mathrm{~mm}$. The coupling of a Frelon CCD camera to an optical system (indirect illumination) was equivalent to using a detector with a pixel size of $0.8 \mu \mathrm{m}$ (effective pixel size). From these two images, the wave front was numerically reconstructed [15] and fitted to an ellipsoid. The same procedure was repeated after insertion of either a dynamically bent mirror or a monolithic focusing mirror. This time the effective pixel size was of $5.8 \mu \mathrm{m}$, the membrane was situated around the focal point of the mirror and $\Delta l=120 \mathrm{~mm}$. The calculated values for the wave front curvature are presented in Table I; they are in good agreement with the experimental conditions.

As phase information can be collected for every pixel, $2 \mathrm{D}$ phase imaging becomes realizable. Phase imaging is of great interest for a sample with low absorption, e.g., made of light materials, or a sample made of different materials with similar attenuation coefficients. For this experiment, the scheme in Fig. 2(a) was used with the sample placed on
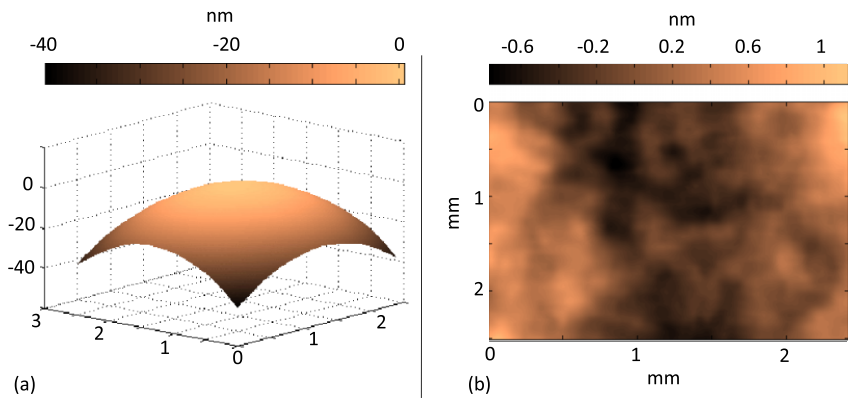

FIG. 3 (color online). (a) Reconstruction of the ESRF BM05 wave front after double reflection on $\mathrm{Si}(111)$ monochromator. (b) Wave front departure from a perfect spherical wave front. 
TABLE I. Comparison between direct length measurements and their determination by the present technique.

\begin{tabular}{lc}
\hline \hline & Calculated distance \\
\hline Direct beam $40.5 \mathrm{~m}$ & $40.7 \mathrm{~m}$ \\
Bent mirror $f=350 \mathrm{~mm}$ & $352 \mathrm{~mm}$ \\
Monolithic mirror $f=60 \mathrm{~mm}$ & $60.5 \mathrm{~mm}$ \\
\hline \hline
\end{tabular}

a translation stage and a fixed cellulose acetate membrane with a pore size of $0.8 \mu \mathrm{m}$ located $600 \mathrm{~mm}$ downstream to generate the speckle pattern. The $2 \mathrm{D}$ detector arrangement had an effective pixel size of $0.8 \mu \mathrm{m}$ and was placed $\Delta l=$ $940 \mathrm{~mm}$ downstream from the membrane. The sample situated at a distance of $40.5 \mathrm{~m}$ from the source, consisted of a poly(methyl methacrylate) (PMMA) cone introducing a phase gradient. The cone radius was $4 \mathrm{~mm}$ and the opening angle $\vartheta=140 \mathrm{deg}$. The complete field of view of the detector was illuminated. The tip of the cone and the detector were both centered with respect to the beam. Sequentially, a first exposure was acquired with the sample present; a second one was acquired after sample removal. The displacement vectors were then calculated for every pixel and the wave front reconstructed. Absorption, estimated to be $\beta \approx 10^{-10}$, had no effect; nevertheless the displacements of the speckle allowed a perfect reconstruction of the cone (Fig. 4, Supplemental Material [16]). At a distance of $940 \mathrm{~mm}$, the radial displacements of the speckle subsets in the cone, equal to 0.41 pixel, correspond to an angular deviation of $0.35 \mu \mathrm{rad}$. From the expression linking $\delta$ to the wave front gradient $\frac{\partial W\left(x_{i j}, y_{i j}\right)}{\partial x}=$ $\delta \tan ((\pi-\vartheta) / 2)$, we obtain $\delta=9.6 \times 10^{-7}$ which is in very good agreement with the refractive term of the optical index for PMMA at $17 \mathrm{keV}$ [17].

When imaging a low absorbing sample, one can get direct access to the absorption map by simply dividing the two images recorded. However, an important point to take care when imaging strongly absorbing sample or when using very low flux, is to have a minimum of counts per pixel to ensure trustable correlation calculations in the DIC algorithm. Moreover, one has to be aware of the size limit
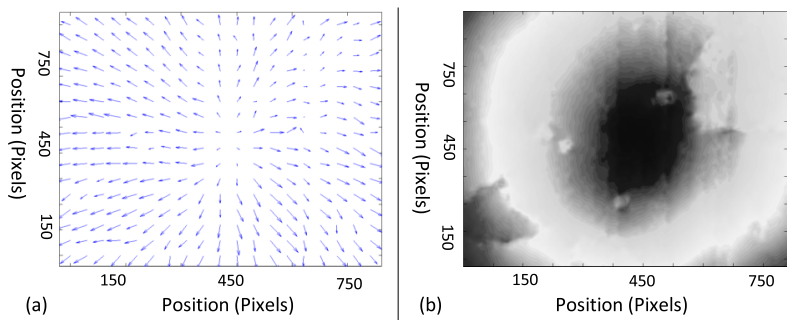

FIG. 4 (color online). Displacement vector field at center (a) and phase-map reconstruction of the cone sample (b). The blurs in the phase map correspond to phase accidents that are due to the presence of micro air bubbles in the glue of the sample holder. of the phase objects that can be resolved; at the position of the membrane, the features of the wave front need to be larger than the speckle grains to not disturb too strongly the speckle pattern. A simple way to overcome this limit and gain resolution is to insert an optical focusing element into the beam and adjust the membrane and sample positions to obtain the required speckle size and sample features through magnification.

Since the beam passes through the membrane with almost no absorption and only one exposure is required for the sample, the sketch shown on Fig. 2(a) is dose efficient, therefore suitable to the analysis of biological tissues for which the dose absorbed is an issue (Supplemental Material [16]).

In conclusion, we have exposed the basis and some illustrations of an efficient technique for accurate quantitative two-dimensional phase sensing of a partially coherent X-ray beam. The presented examples open perspectives for the XST method at synchrotron and X-FEL sources, for instance in bioimaging and inverse ray tracing. The optical simplicity of the experimental configuration may seem to be tempered by the extensive need for computing time. This is actually not a true limitation considering that the used algorithms are perfect candidates for parallel implementations on, for example, some of the new graphics processing unit clusters available on the market.

We wish to thank P. Chavel, J.-Y. Massonnat, T. Weitkamp, I. Zanette, L. Graham, A. Simon, J.-P. Vassalli, J. Baruchel, and K. Sawhney for various contributions. S. Bérujon is also grateful to ESRF and Diamond Light Source for the financial support.

*Present address: SESO, 305 rue Louis Armand, CS 30504, 13593 Aix-en-Provence cedex 3, France.

[1] C. David, B. Nohammer, H. H. Solak, and E. Ziegler, Appl. Phys. Lett. 81, 3287 (2002).

[2] I. Zanette, C. David, S. Rutishauser, and T. Weitkamp, AIP Conf. Proc. 1221, 73 (2010).

[3] P. Mercère, M. Idir, T. Moreno, G. Cauchon, G. Dovillaire, X. Levecq, L. Couvet, S. Bucourt, and P. Zeitoun, Opt. Lett. 31, 199 (2006).

[4] K. A. Nugent, T. E. Gureyev, D. F. Cookson, D. Paganin, and Z. Barnea, Phys. Rev. Lett. 77, 2961 (1996).

[5] T. Weitkamp, A. Diaz, C. David, F. Pfeiffer, M. Stampanoni, P. Cloetens, and E. Ziegler, Opt. Express 13, 6296 (2005).

[6] M. Dierolf, A. Menzel, P. Thibault, P. Schneider, C. M. Kewish, R. Wepf, O. Bunk, and F. Pfeiffer, Nature (London) 467, 436 (2010).

[7] P. Cloetens, W. Ludwig, J. Baruchel, D. Van Dyck, J. Van Landuyt, J. P. Guigay, and M. Schlenker, Appl. Phys. Lett. 75, 2912 (1999).

[8] B. Pan, K. Qian, H. Xie, and A. Asundi, Meas. Sci. Technol. 20, 062001 (2009).

[9] R. Cerbino, L. Peverini, M. A. C. Potenza, A. Robert, P. Bösecke, and M. Giglio, Nature Phys. 4, 238 (2008). 
[10] R. Cerbino, Phys. Rev. A 75, 053815 (2007).

[11] J.W. Goodman, Speckle Phenomena in Optics: Theory and Applications (Roberts and Company Publishers, Greenwood Village, 2006), 1st ed.

[12] W. Zou, K. P. Thompson, and J. P. Rolland, J. Opt. Soc. Am. A 25, 2331 (2008).

[13] R. Coisson, Appl. Opt. 34, 904 (1995).

[14] E. Ziegler, J. Hoszowska, T. Bigault, L. Peverini, J. Y. Massonnat, and R. Hustache, AIP Conf. Proc. 705, 436 (2004).
[15] W. H. Southwell, J. Opt. Soc. Am. 70, 998 (1980).

[16] See Supplemental Material at http://link.aps.org/ supplemental/10.1103/PhysRevLett.108.158102 regarding the cases of phase imaging of the cone and of another cartilage sample. Photon count statistics for these study cases are also given.

[17] B. L. Henke, E. M. Gullikson, and J. C. Davis, At. Data Nucl. Data Tables 54, 181 (1993). 